\title{
Smart Cycle Share System
}

\author{
Akash Vatane ${ }^{1}$, Mayur Yadav ${ }^{2}$, Janardan Narvate ${ }^{3}$, Dr. Mrs. S.D. Joshi ${ }^{4}$ \\ Student, E\&TC, P.V.P.I.T. Pune, India ${ }^{1,2,3}$ \\ E\&TC, P.V.P.I.T. Pune, India ${ }^{4}$
}

\begin{abstract}
This project aims to present microcontroller based security to the bicycle and made the habit to people to use the bicycle as a mode of transportation. Many people in India tried to find out the problems for reducing traffic congestion and pollution, but the easiest way to reduce that problems is to use bicycle in our daily use. Whenever the transportation is not good it makes burden to health and health may make a burden on economy. 'Smart Cycle Share System' project makes people to take the cycle from one station as their transport vehicle and submit the cycle to the station which is nearer to their destination address.
\end{abstract}

Keywords: GSM, RFID Reader, GPS, DC MOTOR.

\section{INTRODUCTION}

Cycling is the easiest and environmentally friendly mode of transport. In India, increase in population is one of the major issues. But with the increased population, the use of vehicles are also increased. The reason behind increasing the vehicles is improper use of social vehicles such as buses and trains etc. Because today's fast generation is very serious about the timings, and due to improper maintenance of buses they are unable to reach at their destination. So people use their private bikes and cars for transportation. This increased in vehicles increases the sound pollution. According to national sound rate, the sound rate should be lesser than 65 decibels. But in India, it goes up to 70 to 90 .

Vehicles may also increases the air pollution. There are more numbers of small rock particles in air and vehicles may produce carbon dioxide and other gases which are harmful to human beings. According to the survey of pollution control committee, the increased in air particles may increase by 90 than last survey. To reduce the pollutions, there are several actions which have been taken by government such as planting, green building, garbage dividation and also to reduce traffic BRT's and Metro's plans are developed. But pollution is not only the one issue the health of people also be in danger because unaware about their health. According to survey of Lancet Research Centre every two person died per day due to diabetes and heart attack. In today's world sitting work should be increased day by day, so these two problems should be increased.

Smart Cycle Share System is the booster dose for all the problems which we have discussed above Means first cycling maintains the health, second is reduce traffic pollution ,third is reducing sound pollution, fourth is reducing air pollution. Vehicles like cars and two wheelers increases the traffic problems but cycle takes less space to park it. So, there is no parking problem while using cycles. In this project we have made a system in which, a person who require a cycle will take cycle from one station. A cycle will be issued on his name and he can enjoy cycling and has to submit the cycle to the destination station. We have added security for the cycle from stole the cycle.

\subsection{RELATED WORK}

For a smart cycle project we are taking the references from three papers. In [1], Ewan Vaughan and Xun Wang develop a mobile app called Social Cycle which purpose is to encourage users to start cycling and to increase the number of trips. From [1] we are taking cycle issuing concept by using RFID Reader. In[2], João C. Ferreira made they develop a mobile application which provide information to cyclist related with the electric bicycle. From [2] we are storing the information of cyclist who issued the cycle from either main system or sub system. In [3], Pradip Suresh Mane and Vaishali Khairnar, in their system, the bus is tracked by the GPS over the intended path. From [3] we also use GPS to track the cycle for security purpose. The project aims in designing a Smart Cycle. We use the Pic microcontroller for controlling and monitoring purpose. By using RFID reader first of all we issue the cycle from source station. For security purpose of the cycle we develop the navigation system by referring [3].We also add the auto locking system for security purpose this idea comes from sky lock system.

\subsection{EXPLAINATION USING BLOCK DIAGRAM}

Smart cycle project consist of three various blocks :-

(1) Main system block

(2) Sub-System block

(3) Cycle system block 
Main system and sub-system are having same function. Only the difference is that the main system having initial parking space and sub-system can park anywhere other than initial parking space. They can be used as master or slave. Main system can be master or slave and the sub-system can also be master or slave. Main system consist of various components like PIC microcontroller, keys, LCD, GSM, RFID reader. The sub-system also has same components. Cycle system consists of PIC microcontroller, GSM, LCD, and two DC motors. One act as a break motor which provide break to the cycle and the other one is to provide the lock to the cycle .The system consist of RFID reader which is used for issuing the Bicycle. It also consists of auto locking system by using dc motor.

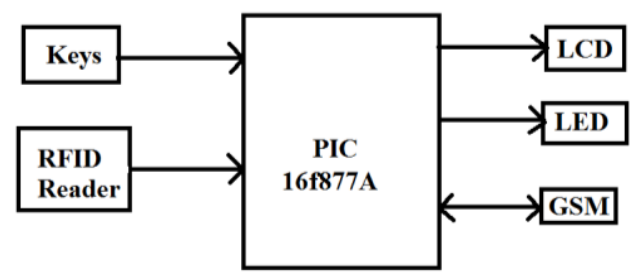

Fig 1.Block diagram of main system

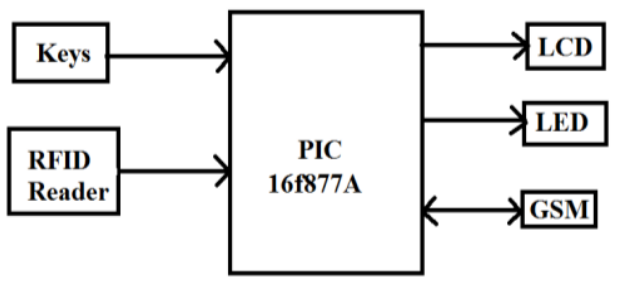

Fig 2.sub System

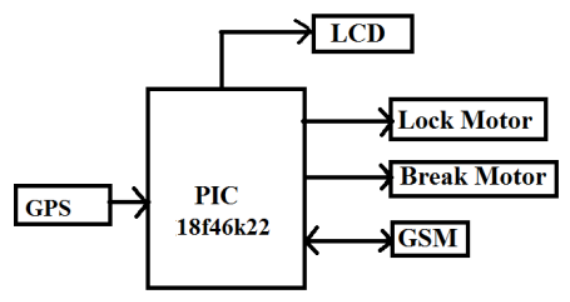

Fig 3.Cycle System

A person can issue cycle from main system by using RFID Reader. We are using GSM for serial communication. The message of 'cycle issued from main system' is delivered to cycle system from GSM of main system. When message arrived from main system the cycle system is unlocked. We are making delay concept so to start the time of cycle, we make additional function of key press. Whenever key is pressed time of cycle start, then what is the delay concept? We make certain time delay to cycle system .If cycle is not arrived to its destination system that is (sub system) at certain time cycle is braked and gets locked. And if cycle is arrived in right time, cycle can be again reissued. Then what is breaking and locking system? We add this feature for cycle security, whenever cycle not arrived to sub system in certain time, sub system will send message to break the cycle and then lock it.

The description of each block in block diagram is as below

\section{EXPERIMENTATION}

(i) PIC-16F877A $\mu \mathrm{C}$ :-The PIC microcontroller PIC16f877a is one of the most renowned microcontrollers in the industry. This controller is very convenient to use, the coding or programming of this controller is also easier. One of the main advantages is that it can be write-erase as many times as possible because it use FLASH memory technology. It has a total number of 40 pins and there are 33 pins for input and output. PIC16F877A is used in many various PIC microcontroller projects. PIC16F877A also have many application in digital electronics circuits.1 serial half duplex port (RC7 (Rx.) -RC6 (Tx.)). Interrupts (rb0 (int0)- rb1 (int1)).Inbuilt I2C bus (RC3 (SCl) - RC4(SDA))Inbuilt SPI bus (ss,sdi,sck,cs). Maximum operating frequency is $20 \mathrm{MHz}$.Flash program memory (14 bit words), 8KB.Data memory (bytes) is 368.EEPROM data memory (bytes) is 256.5 input/output ports. 3 timers. 2 CCP modules. 2 serial communication ports (MSSP, USART). PSP parallel communication port.10bit A/D module ( 8 channels). 
(ii) LCD:-A liquid-crystal display (LCD) is a flat-panel display or other electronically modulated optical device that uses the light-modulating properties of liquid crystals. Liquid crystals do not emit light directly, instead using a backlight or reflector to produce images in color or monochrome. LCDs are available to display arbitrary images (as in a general-purpose computer display) or fixed images with low information content, which can be displayed or hidden, such as preset words, digits, and 7-segment displays, as in a digital clock.LCD shows how much cycles are delivered and how much cycles are submitted.

(iii) GSM:-GSM (Global System for Mobile Communications), is a standard developed by the European Telecommunications Standards Institute (ETSI) to describe the protocols for second-generation (2G) digital cellular networks used by mobile phones, first deployed in Finland in July 1991.As of 2014 it has become the de facto global standard for mobile communications - with over $90 \%$ market share, operating in over 219 countries and territories.2G networks developed as a replacement for first generation (1G) analog cellular networks, and the GSM standard originally described as a digital, circuit-switched network optimized for full duplex voice telephony. In this project they are used to send message to the different stations according to the situations.

(iv) RFID Reader:- Radio Frequency Identifier RFID Reader Module, are also called as interrogators. They convert radio waves returned from the RFID tag into a form that can be passed on to Controllers, which can make use of it. RFID tags and readers have to be tuned to the same frequency in order to communicate. RFID systems use many different frequencies, but the most common and widely used \& supported by our Reader is $125 \mathrm{KHz}$. An RFID system consists of two separate components: a tag and a reader. Tags are analogous to barcode labels, and come in different shapes and sizes. The tag contains an antenna connected to a small microchip containing up to two kilobytes of data.In this Project they are used to take the entries of different cyclist users.

(v) GPS:-A GPS tracking unit is a device, normally carried by a moving vehicle or person, that uses the Global Positioning System to determine and track its precise location, and hence that of its carrier, at intervals. The recorded location data can be stored within the tracking unit, or it may be transmitted to a central location database, or Internetconnected computer, using a cellular (GPRS or SMS), radio, or satellite modem embedded in the unit. This allows the asset's location to be displayed against a map backdrop either in real time or when analyzing the track later, using GPS tracking software. Data tracking software is available for smart phones with GPS capability .To track the cyclist after lock the cycle, GPS is used.

(vi) PIC18F46K22:- It is 40 pins 8 bit microcontroller. It is having c compiler optimized architecture/instruction set. It has also data EEPROM of 1024 bytes. Program memory of 64kbytes and data memory of 4kbytes. Both program and data memories are linear. It has Low voltage option available for $1.8 \mathrm{~V}-3.6 \mathrm{~V}$ operation. It has two UART for serial communication operation. The main reason to use Pic18 is, it has 2 UARTS, and we need one for GSM and one for GPS.

\section{FLOWCHART}

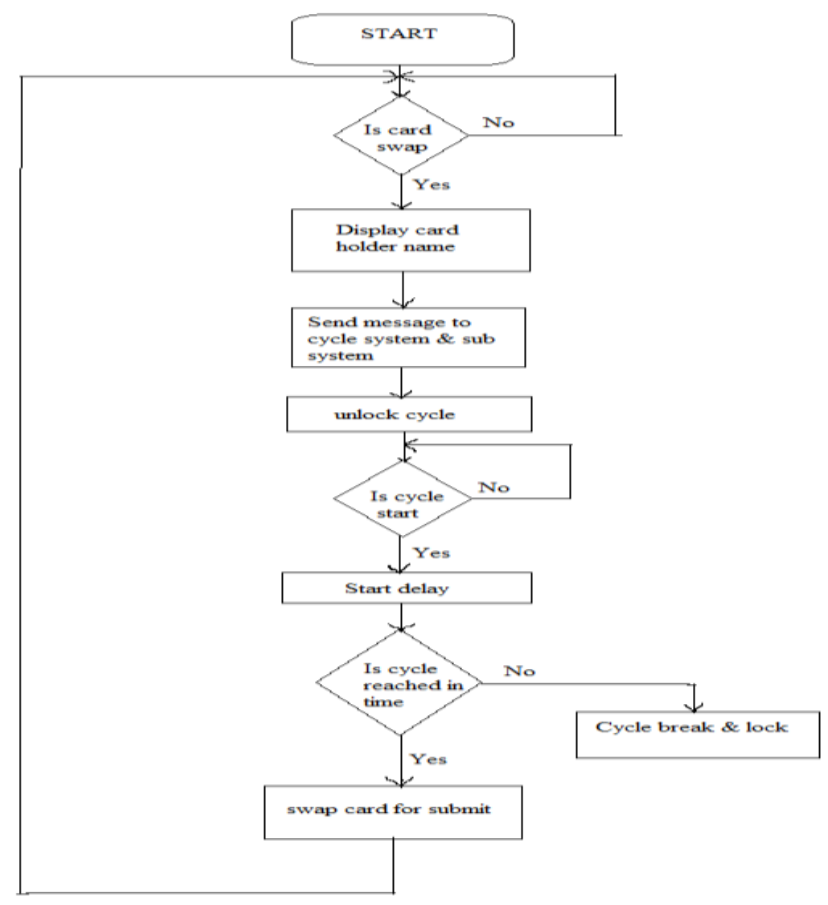

Fig 4. Operational Flow Chart 


\section{ACTUAL OUTPUT}

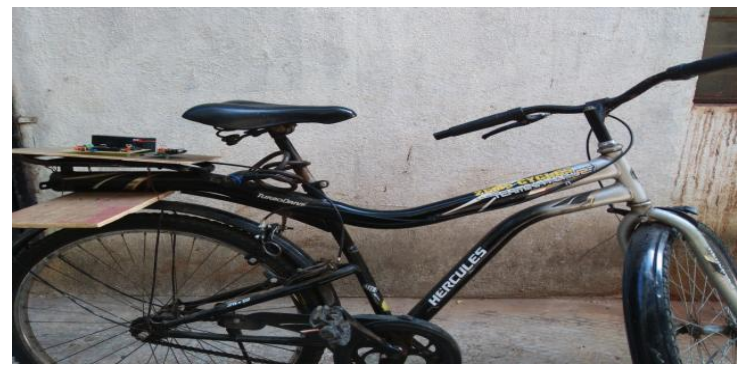

Fig 5. Actual cycle with Cycle System

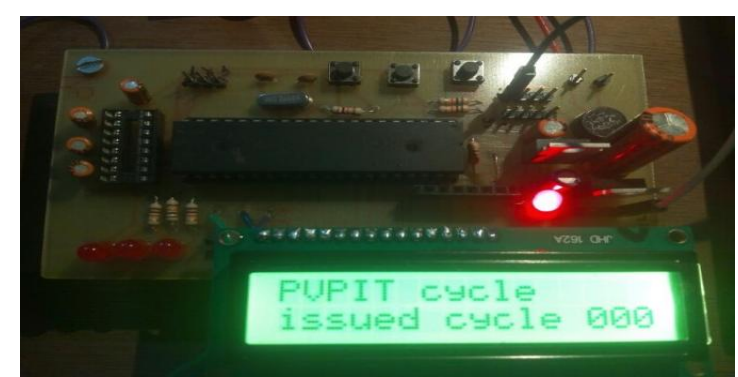

Fig 6. Actual output when no cycle issued

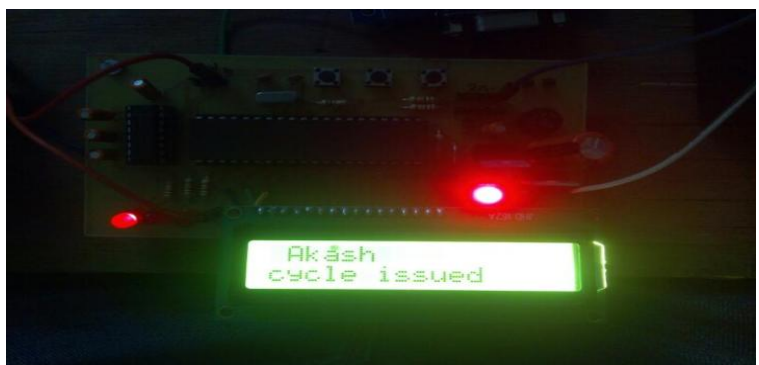

Fig 7. Actual output when one cycle issued

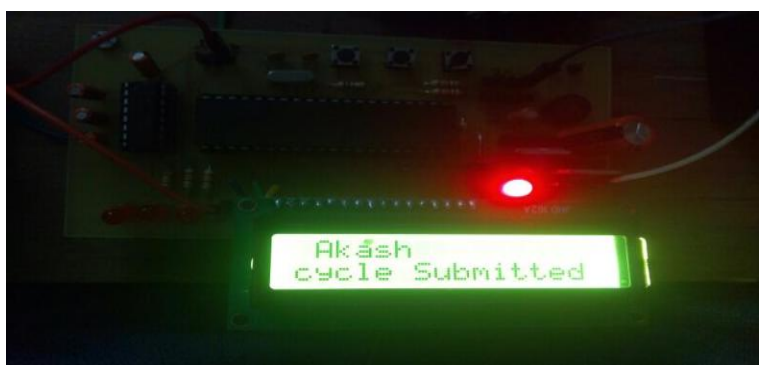

Fig 8. Actual output when one cycle submitted

\section{CONCLUSION}

In this project, we have successfully designed a smart cycle share system using GSM and GPS technology. This project made habit to human being to use the cycle on pay and use basis. While cycle is not reached at the destination the cycle gets locked and tracked by our design. Thus, this project is made eco-friendly and sustainable mode of transport to reduce traffic congestion and developed a positive addiction.

\section{REFERENCES}

[1] Karla Felix Navarro, Valerie Gay, Loic Golliard, Benjamin Johnston, Peter Leijdekkers, Ewan Vaughan, Xun Wang, and Mary-Anne Williams at UTS, University of Technology, "SOCIAL CYCLE"Published, Sydney, Australia, in year 2013.

[2] João C. Ferreira, Member, IEEE, Vítor Monteiro, Student Member, IEEE, José A. Afonso, Member, IEEE, João L. Afonso, Member, IEEE, "MOBILE COCKPIT SYSTEM"in year 2015.

[3] Pradip Suresh Mane, Vaishali Khairnar Analysis of IOSR Journal of Computer Engineering (IOSR-JCE) e-ISSN: 2278-0661, p- ISSN: 22788727Volume 16, Issue 2, Ver. XII (), PP 80-82, "BUS TRACKING SYSTEM USING GPS ON SMART PHONES” in Mar-Apr. 2014. 